\title{
Mobile Application for Detecting Vehicle Accident and Tracking System Using GPS/GSM
}

\author{
Mr. Shubham Ingle ${ }^{1}$, Miss. Ankita Shendkar ${ }^{2}$, Mr. Sanjay Chavan ${ }^{3}$, Prof. Avinash Palave ${ }^{4}$ \\ Student, Comp Dept, KJEI's Trinity College of Engineering and Research, Pune, India ${ }^{1,2,3}$ \\ Guide, Comp Dept, KJEI's Trinity College of Engineering and Research, Pune, India ${ }^{4}$
}

\begin{abstract}
Accident is the authority thread for the people's life which causes a very harmful or dead full. The automotive companies have made lots of progress in reducing this thread, but still the probability of harmful effect due to an accident is not reduced. Contravention of speed is one of the fundamental reasons of accident. Therewithal, external pressure and change of angle with road surface blameworthy for this mistake. As possible as the emergency service could disclose about an accident, the more the effect would be reduced. For this purpose, we developed an Android application that detects an accidental situation and sends emergency alert message to the nearest police station and health care centre. This application is integrated with an external pressure sensor to extract the noticeable force of the vehicle body. It will calculate speed and change of tilt angle with GPS and accelerometer sensors respectively on Android phone. And checking conditions, this application also capable of reducing the rate of false alarm.
\end{abstract}

Keywords: Detecting Vehicle Accident, Sensors, GPS module, GSM module, Intelligent Transport System (ITS)

\section{INTRODCTION}

The world has shown an enhancement in its production over the recent years. Millions of vehicles are being produced annually. But along with the accident rates are also getting significantly increased. As a result, even the optimistic nature of people has become worried while going outside. Most of the accidents occur due to human negligence, such as reckless driving, lack of good infrastructure, etc. An immediate rescue process after an accident can be considered as a tightrope walk between life and death. Any fractional time delay of arriving medical help can cost the life of the victims. A study by Virtanen et al. shows that $4.6 \%$ of the fatalities in accidents could have been prevented only in Finland if the emergency services could be provided at the place of the accident at the proper time. As such, an efficient automatic accident detection with an automatic notification to the emergency service with the accident location is a prime need to save the precious human life. As smart phones become such an important part of our life, it is feasible to use smart phones in a post-accident fatality prevention system. Our application uses the GPS receiver in phone to detect the rapid change of deceleration that occurred at accident time. By detecting these three conditions as accident detection, this android app send the accident location for emergency help. An emergency switch option also added to this app which provides a chance to driver for sending alert message without checking accident detection condition.

Therefore, our contribution can be listed as follows:

- Construction of an efficient automated vehicle accident detection system using Android.

- Develop a framework for reducing false alarm of vehicle accident detection.

- Dispatch automatic emergency accident alert message to relative, nearest police station and hospital. We have discussed on some relevant papers in section II. In section III we have chronicled the system functionality with technical details.

\section{LITERATURE SURVEY}

Title: Safe Driving: A Mobile Application for Detecting Traffic Accidents

Author: Samah Jamal, Houssam Zeid, EliasYaacoub

Description: about the place and time of the accident in addition to some personal information about the driver (e.g., full name, telephone number, number of a relative, blood type, plate number, etc.). Furthermore, it detects car accidents through an analytical model that integrates measurement data collected from the mobile phone (e.g., on toring the variation of speed, acoustic waves, and vibration waves). 
Vol. 7, Issue 12, December 2018

Limitations: To avoid false alarms and the generation of unnecessary reports to the traffic center, the occurrence of an accident is confirmed if the driver did not respond to a question asked by the application to confirm that an accident took place: If the user does not cancel the alert, it is assumed genuine and the report is sent. In the opposite case, the user would be safe since no accident occurred and he/she can respond to the question and cancel the alert thus preventing a crash report from being sent.

Title: -Real Time Traffic Accident Detection System using Wireless Sensor Network.

Autor: - Hossam M. Sherif, M.Amer Shedid, Samah A. Senbel, International Conference of Soft Computing and Pattern Recognition

Description: - Automatic vehicle accident detection is a life-saving application that is vital in today's high-speed motorways. In case of motorway accidents, notification to the proper authorities must be done efficiently and expediently. The main objective of this paper is to create a Real Time Traffic Accident Detection System (RTTADS) using Wireless Sensor Network (WSN) and Radio-Frequency Identification (RFID) Technologies. This paper explains the hardware prototype setup for RTTADS, the algorithms used, the advantages and the limitations of the entire system. Also, the configuration of the setup and application software is elaborated. Sensors installed in a vehicle detect the accident's location, the vehicle's speed just before the accident and the number of passengers in the vehicle. The sensors then send an alert signal to a monitoring station. The monitoring station, in turn, tracks the location where the accident has occurred and directs casualty alert to the authorities concerned.

Title: - Intelligent System for Vehicular Accident Detection and Notification.

Author: - Bankar Sanket Anil, Kale Aniket Vilas, Prof. S. R. Jagtap

Description: -Road accidents are a human tragedy. They involve high human suffering and monetary costs in terms of untimely deaths, injuries and loss of potential income. There are so many new techniques such as Antilock Braking System (ABS), Adaptive Cruise Control (ACC), Anti Collision System (ACS) to avoid accidents and in spite of all this, such large number of accidents takes place. Hence this paper presents a system which gives an idea about what can be done to provide medical help and other facilities after accident as soon as possible. Accident can be detected using flex sensor and accelerometer, while location of accident will be informed to desired persons such as nearest hospital, police and owner of vehicle through SMS sent using GSM modem containing co-ordinates obtained from GPS along with time of accident and vehicle number. Camera located inside vehicle will transmit real time video to see current situation of passengers inside vehicle. Thus, this paper emphasizes on post-accident system for detecting and informing about it. Simulation result on hyper terminal is also presented in this paper.

\section{PROPOSED SYSTEM}

In Proposed system we are using the sensors, GPS and GSM modules. And We also make Android application and, in that user, can register the application. And registration successful then Login the application. Similarly, admin can login the application. So, admin using application see the user details. so, hardware module is following:

1. Sensors: - In that two type of a sensor are used:

SW420: This sensor is used for detection of vibration.

ADXL335: This sensor is used for measure the static acceleration of gravity in tilt-sensing applications, as well as dynamic acceleration resulting from motion, shock, or vibration.

2. GPS module

It is a global navigation satellite system that gives geolocation and time information to GPS. A GPS receiver anywhere on the Earth where there is an open line of sight to four or more GPS satellites. GPS systems are extremely adaptable and can be found in almost any sector. GPS systems are used in military applications and by emergency band to locate people in need of assistance. It has three parts: satellites, ground stations, and receivers. And receiver calculates the distance from four or more satellites, it knows exactly where you are.

\section{GSM module}

Global System for Mobile communication is a digital mobile telephony system that is widely used in world. GSM uses a variation of time division multiple access and is the most widely used of the three digital wireless telephony 
technologies and General Packet Radio Services is a packet-based wireless communication service that promises data rates from 56 up to $114 \mathrm{Kbps}$ and continuous connection to the Internet for mobile phone GPRS provides quick session setup and fast data transmission speed. GPRS base stations and support nodes use Internet Protocol for relaying messages.

\section{SYSTEM ARCHITECTURE}

Following diagram is our system's architecture diagram: In system architecture Mobile devices, especially Smart phones have been deployed as floating traffic probes and sensors in many applications, both academically and commercially. These applications include road conditions survey, traffic conditions monitoring and accident detections. All of these abilities are essential to an Intelligent Transport System (ITS), which aims to reduce traffic congestion and enhance traffic safety.

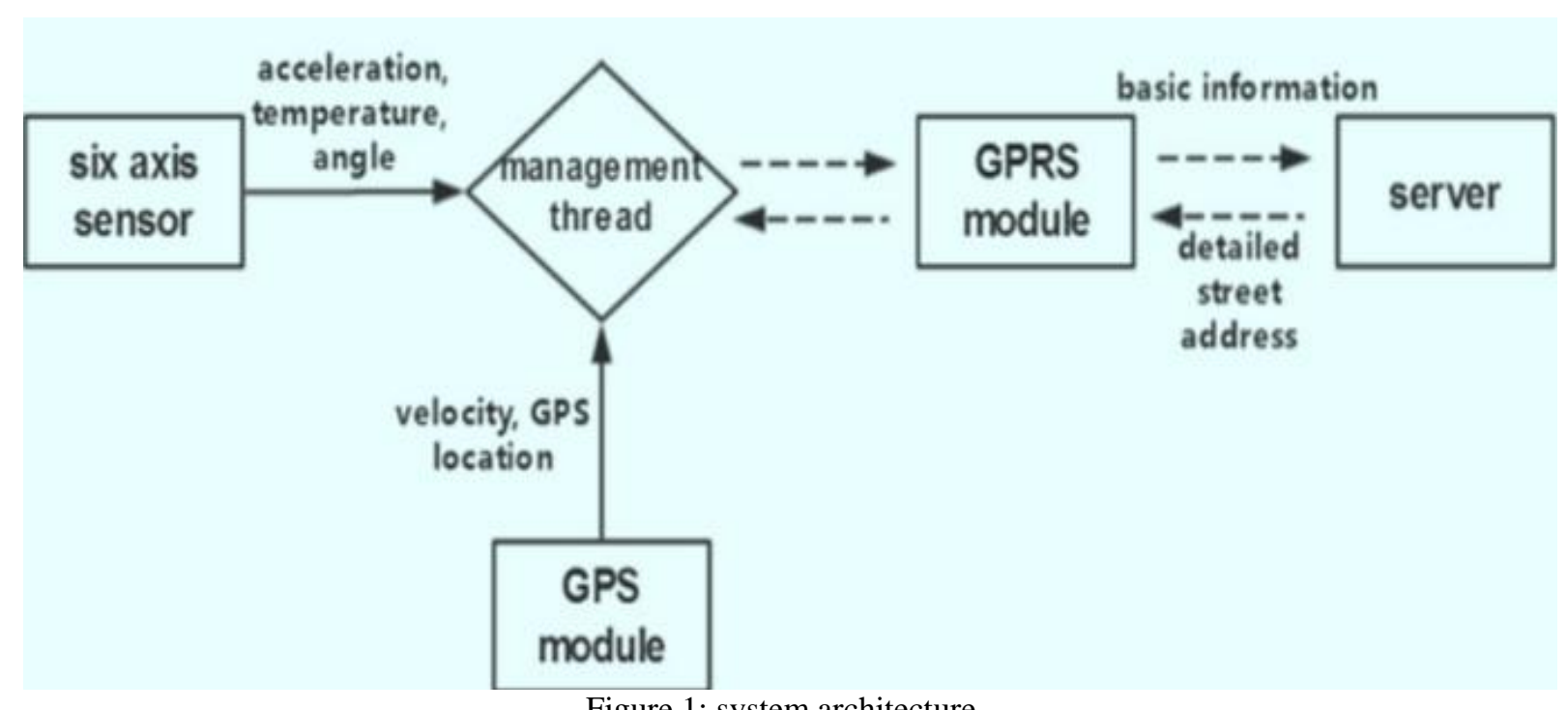

Figure 1: system architecture

Real-time traffic accident prediction focuses is change of traffic conditions, while traffic incident detection studies are concerned with the change of traffic conditions after an incident happened. However, the performance of these detection and prediction system is greatly restricted by the number of monitoring sensor, available fund, the algorithms used to confirm an accident, weather, traffic flow etc. There are possibilities of false alarm in the system and also does not guarantee the occurrence of an accident. S. Amin et al. proposed a method of accident detection using GPS, GPRS and GSM.

Accident Detection We have considered three parameters to detect a situation as an accident. As we know, when an accident occurs velocity of vehicle decrease rapidly. But in this application, we do not detect the situation as an accident, only when the speed decreases rapidly.

\section{METHODOLOGIES}

\section{A. Accident Detection}

In case 1 , if the speed of vehicle dropped down rapidly and a change of tilt angle with road surface increases much, then it detects as an accident. Actually, it means when the threshold values of speed and change of tilt angle of vehicle exceed, then our application considers the situation as an accident. And, this time two flag bits change and flag number tum into '023'. In case 2, when the threshold values of speed and pressure of vehicle exceed, it considers the situation as an accident.

\section{B. Alarm and Emergency Alert Message}

When any one of our proposed accident detection method happen, then an alarm arises. User can press 'Cancel' button and no emergency alert message will send. This kind of situation is considered as a false alarm. Otherwise, an emergency alert message will send to user's emergency contact number, nearest police station and hospital. 


\title{
IJARCCE
}

\section{International Journal of Advanced Research in Computer and Communication Engineering}

\author{
Vol. 7, Issue 12, December 2018
}

\section{CONCLUSION}

In this paper, we have shown that road accident can be detected efficiently by using some particular parameters. Our proposed approach capable of deciding whether a situation is an accident or not and if so, then immediately traces nearest police station as well as hospital and send emergency alert message for help. we presented a fusion of infrastructure-based system with an ad-hoc communication network which enables a wide variety of use cases for enhancing traffic safety. Due to the portable and stable features, collision sensor device can embed almost every vehicle, as well as the customer application.

\section{REFERENCES}

[1]. Zhengxu Hou, Yiping Chen“A Real Time Vehicle Collision Detecting and Reporting System Based on Internet of Things Technology”2017.

[2]. Samah Jamal, Houssam Zeid, EliasYaacoub "Safe Driving: A Mobile Application for Detecting Traffic Accidents." 2018

[3]. Ravi K Kodali, Shubhi Sahu"MQTT Based Vehicle Accident Detection and Alert System"2017.

[4]. Shadman Sakib, Mohammad Sayem Bin Abdullah "GPS-GSM based Inland Vessel Tracking System for Automatic Emergency Detection and Position Notification." 2016.

[5]. Adnan Bin Faiz, Ahmed Imteaj, " Smart Vehicle Accident Detection and Alarming System Using a Smartphone " 2015.

[6]. Asad Ali, Mohamad Eid "An Automated System for Accident Detection." 2015

[7]. N. Watthanawisuth, T. Lomas and A.Tuantranont "Wireless Black Box Using MEMS Accelerometer and GPS Tracking for Accidental Monitoring of Vehicles" 2012

[8]. P. Bartłomiej, "Performance Evaluation of Road Traffic Control using a Fuzzy Cellular Model “, 2011.

[9]. Md. Syedul Amin, Jubayer Jalil , M. B. I. Reaz "Accident Detection and Reporting System using GPS, GPRS and GSM Technology" 2012

[10]. Amit Meena, Srikrishna Iyer, Monika Nimje,Saket JogJekar, Sachin Jagtap,Mujeeb Rahman "Automatic Accident Detection and Reporting Framework for Two Wheelers" 2014. 